\title{
Zur Kenntniss der pflanzlichen Oxydasen.
}

\author{
Von \\ Dr. B. Slowtzoff.
}

(Aus dem physiologisch-chemischen Laboratorium der St. Petersburger militärmedicinischen Akademie.)

(Der Redaction zugegangen am 11. October 1900.)

Bei der Untersuchung der Oxydationsvorgänge in den thierischen und pflanzlichen Organismen fand man eine Reihe organischer Substanzen, die den Sauerstoff auf andere chemische Verbindungen auch in vitro übertragen können; so theilte Schönbein schon im Jahre 1848 mit, dass verschiedene frische thierische Gewebe und die Säfte vieler Pflanzen Wasserstoffsuperoxyd zerlegen und Guajactinctur blau färben, dass sie aber diese Fähigkeiten durch Kochen verlieren. Diese Erscheinung blieb lange ohne Erklärung, bis Bertrand, ${ }^{1}$ ) der die Versuche des japanischen Chemikers Hikorokuro Yoshida ${ }^{2}$ wiederholte, fand, dass man auch aus dem Harz des Lackbaums eine Substanz isoliren kann, die einen andern Bestandtheil des Lackbaumharzes, das Laccol, energisch oxydirt. Doch werden viele andere Körper, wie Hydrochinon, Pyrogallol, Guajacolsäure, einige Polyphenole und Oxyhydrate der Phenole, auch einige Aether und Amine der aromatischen Reihe durch die von Bertrand entdeckte Substanz oxydirt; sie wurde Laccase genannt und kann als Typus eines oxydirenden Fermentes gelten. Die Oxydation geht am besten bei neutraler, aber auch bei schwach saurer Reaction vor sich, 50\%iger Aethyl- oder Methylalkohol ist ohne Einfluss auf den Ablauf derselben.

Bertrand ${ }^{3}$ ) konnte 3 Präparate von Laccase darstellen; das erste, aus dem Harze des Lackbaums gewonnen, war aber nicht rein. Es enthielt ungefähr 86,77\% Gummi (Araban und Galactan), 7,40\% Wasser (durch Trocknen bei $120^{\circ}$ bestimmt), 5,58\% an Mangan reiche Asche und etwa 2,5\% Eiweiss, nach dem Stickstoffgehalt berechnet. Das zweite Präparat, aus Annamlack gewonnen, enthielt 7,2\% $\mathrm{H}_{2} \mathrm{O}$ (bei

Hoppe-Seyler's Zeitschrift f. physiol. Chemie. XXXI. 
$110^{\circ}$ getrocknet), 4,6\% Asche und 0,117\% Mangan (colorimetrisch bestimmt); das dritte wurde aus Medicago sativa isolirt und hatte den enormen Aschegehalt von 45,2\%.

Durch weitere Untersuchungen fanden Bertrand ${ }^{4}$ ) und Bourquelot, ${ }^{5}$ ) dass die Laccase in der Pflanzenwelt sehr verbreitet war, dabei konnte Bertrand ein zweites oxydirendes Ferment pflanzlichen Ursprungs isoliren, das wegen seiner Fähigkeit, Tyrosin zu oxydiren, Tyrosinase genannt wurde. Gairod ${ }^{6}$ ) entdeckte 1895 im Wein ein Ferment, dass das Sauerwerden desselben verursachte; Martinaud, ${ }^{10}$ ) Cazeneuve, ${ }^{7}$ ) Laborde ${ }^{8}$ ) und Lagatu ${ }^{9}$ ) konnten bei einer Wiederholung der Versuche die Richtigkeit der Gairod'schen Angaben bestätigen. 1897 isolirte Tolomei ${ }^{11}$ ) aus dem Olivenöl ein Ferment, welches das Sauerwerden des Oels verursacht, die Olease; Boutroux ${ }^{12}$ ) konnte aus dem Mehl ein oxydirendes Ferment darstellen, das Oxydin. So wurden also in den letzten Jahren 5 pflanzliche oxydirende Fermente isolirt, ohne hier von den 0xydasen thierischen Ursprungs zu reden, die von Abelous und Biarnès, Spitzer, Salkowski, Petry u. A. entdeckt und beschrieben wurden.

Da die Isolirung der oxydirenden Fermente viele Schwierigkeiten bietet, so ist ihre chemische Natur noch nicht Gegenstand eingehender Studien gewesen; Bertrand glaubte, sie seien organische Manganverbindungen, die sich leicht dissociiren, doch gründet sich seine Theorie mehr auf Analogien als auf experimentelle Prüfung. Es ist daher vor allen Dingen wünschenswerth, die pflanzlichen Oxydasen besser zu isoliren und sich mit den Gesetzen ihrer Wirkung und ihren Eigenschaften bekannt zu machen.

\section{Zur chemischen Natur der Oxydasen.}

Als Ausgangsmaterial zur Darstellung der Laccase dienten Kartoffeln und Kohl. Einige Kilo frischer gewaschener Kartoffeln wurden gehackt und zu Brei zerquetscht, dieser mit verdünnter Essigsäure $(0,5-1 \%)$ versetzt, um die Wirkung der Oxydase auf Tyrosin und andere Farbstoff liefernde Körper der Kartoffel zu vernichten. Nach 24 Stunden wurde das 
Extract durch ein Tuch colirt und abfiltrirt. Das klare; röthliche oder gelbliche Filtrat wurde mit Ammonsulfat gesättigt, der so gewonnene Niederschlag, der Eiweisskörper, Farbstoffe und Ferment enthielt, auf einem Filter gesammelt, mehrmals mit kalter gesättigter Ammonsulfatlösung ausgewaschen und wieder in Wasser gelöst. Das Aussalzen mit Ammonsulfat und Auflösen in Wasser wurde 3 bis 4 Mal wiederholt. Die so gewonnene wässerige Lösung der Eiweissstoffe und Fermente der Kartoffeln wurde gegen fliessendes Wasser in einem Pergamentschlauch dialysirt und mit dem 4-5fachen Volumen $95 \%$ igen Aethylalkohols gefällt, der Niederschlag auf einem Filter gesammelt, mit Aether gewaschen und über Schwefelsäure im Exsiccator getrocknet. Nach einigen Wochen wird der Niederschlag (ein gelblich-braunes Pulver) mit destillirtem Wasser extrahirt und man erhält eine wasserklare Lösung, aus der nach monatelangem Stehen (mit Chloroform gesättigt) ein feiner weisser Niederschlag ausfällt, doch ist die Menge desselben kaum zu wägen. Zur Darstellung des reinen Fermentes wird das beschriebene Extract mit dem 5-6 fachen Volumen Aethylalkohol gefällt, der Niederschlag gesammelt und über Schwefelsäure im Exsiccator getrocknet. Die Ausbeute der reinen Laccase ist so klein, dass ich nach jahrelangem Sammeln ungefähr $1 \mathrm{~g}$ der reinen Substanz bekommen habe.

Die Darstellung der Laccase aus dem Kohl ist ganz dieselbe, die Ausbeute ist noch kleiner, aber das Präparat kann schneeweiss erhalten werden, während das aus den Kartoffeln dargestellte immer eine gelbliche Farbe hat.

Die Lösung dieser Präparate gab intensive Biuretreaction, Xanthoprotein-, Millon'sche, Liebermann'sche, Pettenkofer'sche und Adamkiewicz'sche Reaction, ebenso alle Alkaloid- und Fällungsreactionen.

Bei vollständiger Sättigung der Fermentlösung mit Magnesiumsulfat fällt die Laccase nicht ganz aus, Kochsalz und Natriumsulfat fällen sie garnicht, dagegen wird sie bei vollkommener Sättigung mit Ammonsulfat vollständig gefällt. Aethylalkohol fällt sie nur bei einem Gehalt von $90 \%$ aus. 
Der Aschegehalt meiner Laccasepräparate ist sehr klein, die zwei ersten Präparate ergaben:

0,1218 Substanz lieferten 0,0014 Asche $=1,49 \%$

$0,0861>0,0013>=1,51 \%$, im Mittel $1,5 \%$.

Das dritte und vierte Präparat, die noch sorgfältiger dialysirt waren, ergaben:

0,1115 Substanz gaben 0,0011 Asche $=0,987 \%$

$0,5415>0,0314>=0,58 \%$, im Mittel $0,78 \%$.

Die Asche wurde in den über Schwefelsäure getrockneten Präparaten bestimmt, beim Trocknen bei $120^{\circ}$ trat noch ein Wasserverlust von 2,75\% ein. Die Asche enthielt Kalium, Natrium, Calcium und Spuren von Eisen, Phosphor und Mangan wurde in unseren Präparaten nicht gefunden.

In den Präparaten wurde ferner noch der Stickstoff- und Schwefelgehalt bestimmt, der Stickstoff nach KjeldahlWilfarth:

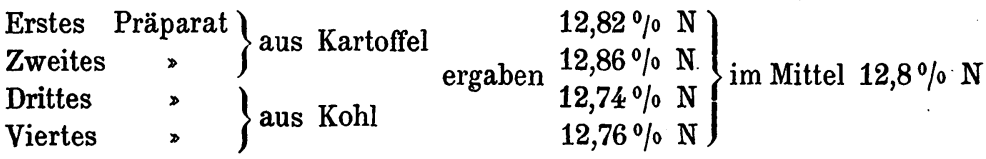

Der Schwefel wurde nach der Verbrennung mit Soda und Salpeter in Form von Baryumsulfat bestimmt:

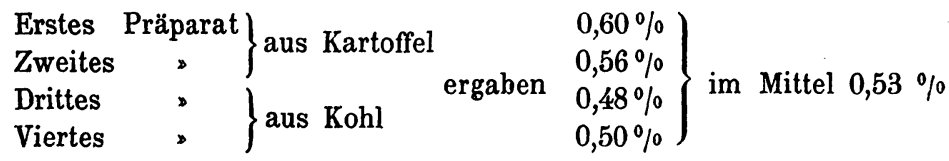

Aus diesen Analysen ergibt sich, dass die Laccase aus Kartoffeln und Kohl eine eiweissartige Substanz $(12,8 \% \mathrm{~N}, 0,53 \% \mathrm{~S}$, alle Eiweissreactionen) und sehr arm an Asche ist. Sie gehört zu den Albuminen und enthält weder Mangan noch Phosphor.

\section{Ueber einige Gesetze der Laccasewirkung.}

Zur Bestimmung der Wirkung der Laccase wählte ich das Röhmann'sche Reagens, und zwar wurde die Menge des entstandenen Farbstoffes colorimetrisch gemessen. Als Einheit diente folgende Lösung: $10 \mathrm{ccm}$. destillirtes Wasser, je 2 Tropfen $2 \%$ iger wässeriger Paraphenylendiaminlösung und 2\%oiger wässe- 
riger Metatoluilenlösung, 2 Tropfen ( $1 / 10 \mathrm{ccm}$ ) $10 \%$ iger Sodalösung, dann wurde zur Mischung ein Tropfen 5\% oiger wässeriger Ferrocyankalilösung beigefügt; die Menge des entstandenen Farbstoffs wurde gleich 100 gesetzt.

Die erste Reihe der Versuche wurde angestellt, um zu erfahren, ob es eine Proportionalität zwischen der Menge des Fermentes und der Menge des entstandenen Farbstoffes gäbe. In einer Reihe von Probirröhren wurden $10 \mathrm{ccm}$. der oben erwähnten Flüssigkeit abgemessen und mit verschiedenen Mengen der Ferment(Laccase)lösung versetzt. Nach einigen Stunden wurde der Farbstoffgehalt colorimetrisch bestimmt.

\begin{tabular}{|c|c|c|c|c|c|c|}
\hline $\begin{array}{l}\text { Nr. des } \\
\text { Ver- } \\
\text { suchs }\end{array}$ & $\begin{array}{c}\text { Die Menge des } \\
\text { Farbstoffs ohne } \\
\text { Ferment }\end{array}$ & $\begin{array}{c}\text { Mit } \\
5 \text { Tropfen } \\
\text { Laccaselös. }\end{array}$ & $\begin{array}{c}\text { Mit } \\
10 \text { Tropfen }\end{array}$ & $\begin{array}{c}\text { Mit } \\
15 \text { Tropfen }\end{array}$ & $\begin{array}{c}\text { Mit } \\
\text { 20 Tropfen }\end{array}$ & $\begin{array}{c}\text { Mit } \\
\text { 25 Tropfen }\end{array}$ \\
\hline 1 & 4,0 & 5,0 & 6,25 & 8,75 & 10,5 & - \\
\hline 2 & 5,0 & 7,25 & 17,0 & 22,0 & 26,0 & 30,0 \\
\hline 3 & 6,0 & 7,0 & 10,0 & 15,0 & 19,0 & 20,0 \\
\hline 4 & 5,0 & 7,25 & 13,0 & 14,5 & 17,0 & 19,0 \\
\hline 5 & 8,0 & 16,5 & 21,7 & 30,0 & 36,0 & 42,0 \\
\hline 6 & 5,0 & 6,0 & 9,25 & 17,0 & 20,0 & 24,0 \\
\hline 7 & 8,0 & 20,2 & 27,0 & 36,0 & 38,0 & 39,0 \\
\hline 8 & 5,0 & 7,75 & 12,0 & 16,0 & 18,5 & 18,5 \\
\hline 9 & 5,0 & 7,0 & 11,0 & 15,0 & 16,0 & 16,5 \\
\hline 10 & 5,0 & 6,25 & $7, \check{0}$ & 8,0 & 11,0 & 16,0 \\
\hline Mittel & 5,6 & 9,02 & 13,47 & 18,25 & 22,20 & 25,0 \\
\hline
\end{tabular}

Setzt man die Menge des Fermentes gleich 1 und die Menge des durch die Fermenteinheit gebildeten Farbstoffs ebenfalls gleich 1, so kann man die gewonnenen Resultate in folgender Tabelle darstellen:

\begin{tabular}{|c|c|c|c|c|c|}
\hline Menge der Laccase ... . . . . . & 1 & 2 & 3 & 4 & $\mathbf{5}$ \\
\hline Menge des entstandenen Farbstoffs ... & 1 & 1,49 & 2,09 & 2,34 & 2,62 \\
\hline $\begin{array}{l}\text { Quadratwurzeln aus der Menge des Fer- } \\
\text { mentes (nach Schütz-Boris off). } .\end{array}$ & 1 & 1,41 & 1,90 & 2,00 & 2,23 \\
\hline
\end{tabular}

Eine direkte Proportionalität besteht also nicht, dagegen entspricht die Menge des gebildeten Farbstoffs den 
Quadratwurzeln aus der Menge des Fermentes. Von Medwedew ${ }^{14}$ ) wurde eine solche Proportionalität ebenfalls für die Leberoxydase constatirt; die pflanzlichen Oxydasen folgen also der Schütz-Borisoff'schen Regel, ebenso wie Pejpsin, Pancreatin, Oleopsin u. s. w. (Walter) ${ }^{15}$ ). In meinen Versuchen hat die Regel so lange Gültigkeit, bis die Menge des Fermentes auf das Vierfache vergrössert ist.

Zweitens wollte ich die Frage lösen, ob die Menge des Röhmann'schen Reagens die Menge des entstandenen Farbstoffs beeinflusst. Es wurden also zu $10 \mathrm{ccm}$. Fermentlösung verschiedene Mengen des Röhmann'schen Reagens gesetzt und nach einigen Stunden die Menge des entstandenen Farbstoffes colorimetrisch bestimmt.

\begin{tabular}{|c|c|c|c|c|c|c|c|}
\hline & $\begin{array}{c}\mathbf{2} \\
\text { Tropfen }\end{array}$ & $\begin{array}{c}4 \\
\text { Tropfen }\end{array}$ & $\begin{array}{c}6 \\
\text { Tropfen }\end{array}$ & $\begin{array}{c}8 \\
\text { Tropfen }\end{array}$ & $\begin{array}{c}10 \\
\text { Tropfen }\end{array}$ & $\begin{array}{c}12 \\
\text { Tropfen }\end{array}$ & \\
\hline $\begin{array}{l}\text { Versuchs- } \\
\text { Nr. }\end{array}$ & \multicolumn{7}{|c|}{$\begin{array}{c}2 \% \text { wässeriger Lösung von Paraphenylen- und } \\
\text { Metatoluilendiamin }\end{array}$} \\
\hline 11 & $5 ; 0$ & 5,1 & 5,0 & 5,0 & 5,0 & 5,2 & $\ddot{\Phi}$ \\
\hline 12 & 5,0 & 4,8 & 5,2 & 5,0 & 5,2 & 4,9 & 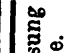 \\
\hline 13 & 5,0 & 5 & 5,4 & 5,6 & 5,2 & 5,1 & \\
\hline 14 & 5,0 & 4,8 & 5,2 & 5,1 & 5,4 & 5,2 & \\
\hline 15 & 5,0 & 4,8 & 5,0 & 5,2 & 5,4 & 5,0 & \\
\hline Mittel & 5,0 & 4,9 & 5,1 & 5,1 & 5,2 & 5,0 & \\
\hline 16 u., 17 & 3,5 & 5,5 & 6,0 & 7,5 & 7,5 & 10,0 & 봉영 \\
\hline 18 u. 19 & 5,0 & 6,5 & 8,0 & 11,0 & 15,0 & 20,0 & 喓 \\
\hline Mittel & 4,3 & 6,0 & 7,0 & 9,3 & 11,3 & 15,0 & 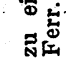 \\
\hline
\end{tabular}

Die Menge des entstandenen Farbstoffs ist also eine Function der Menge des Fermentes, nicht aber der Menge des oxydirenden Reagens. Bei Anwendung eines anorganischen Sauerstoffüberträgers (wie Ferrum sesquichloratum) wird die Menge des entstandenen Farbstoffs proportional der Menge des oxydirenden Reagens: 
In der Litteratur habe ich ein ganz analoges Factum gefunden: Duclaux ${ }^{13}$ ) theilt nämlich in seiner Mikrobiologie mit, dass die Menge der Dextrose, welche durch Invertinwirkung entsteht, eine Function der Menge des Invertins ist. Vielleicht liegt ja hier ein allgemeines Gesetz der Fermentwirkung vor.

MitHülfe der oben beschriebenen colorimetrischen Methode habe ich ferner die Versuche Bertrand's und.Bouquelot's über die Wirkung der Reaction der Flüssigkeit auf die Farbstoffbildung (Oxydation) wiederholt. Wenn nun auch die Reaction einen grossen Einfluss ausübt und eine starke Säure die Thätigkeit des Fermentes vernichten kann, so kann man doch z. B. Kartoffellaccase 24 Stunden mit 0,8-1\% \% iger Essigsäure digeriren, ohne sie zu zerstören. Wenn man die Säure neutralisirt, fängt das Oxydationsvermögen der Laccase wieder an. Die folgende Tabelle zeigt die Stärke der oxydirenden Kraft des Fermentes nach Digeriren mit Säuren verschiedener Concentration.

\begin{tabular}{c|c|c|c|c|c|c|c|c}
\hline \hline Concentration & $0,15 \%$ & $0,25 \%$ & $0,4 \%$ & $0,5 \%$ & $0,8 \%$ & $1,0 \%$ & $1,5 \%$ & $2,0 \%$ \\
\hline Säuren & & & & & & \\
\hline Salzsäure ... & $+1)$ & + & + & + & $\mathrm{Sp}$ & $\mathrm{Sp}$ & $\mathrm{Sp}$ & - \\
Essigsäure ... & + & + & + & + & + & + & + & $\mathrm{Sp}$ \\
Ameisensäure . & + & + & + & + & + & + & $\mathrm{Sp}$ & $\mathrm{Sp}$ \\
Milchsäure... & + & + & + & + & + & + & + & +
\end{tabular}

Durch eine Reihe von Versuchen habe ich ferner festgestellt, dass die Laccase auch nicht durch Pepsinsalzsäure zerstört wird, wenn nur der Säuregehalt in der Flüssigkeit nicht $0,15-0,5 \%$ übersteigt. Kurze Einwirkung der Pancreasverdauung zerstört sie ebenfalls nicht, wie schon Portier gefunden hat, lange fortgesetzte lässt die Kraft des Fermentes allmählich verschwinden.

Die tödtliche Temperatur für die Laccase variirt je nach der Reinheit des. Präparates; die reinsten meiner Präparate

1) + Positiv, - Negativ, Sp Spuren. 
wurden schon bei $50^{\circ}$ zerstört, die aschereichsten erst bei $65-70^{\circ}$.

Aus dieser vorliegenden Arbeit lassen sich folgende Schlüsse ziehen:

1. Die Laccase gehört zu den Fermenten, denn:

1. verliert sie ihre Wirkung bei hohen Temperaturen,

2. ist ihre Wirkung proportional der Quadratwurzel ihrer Menge,

3. ist die Menge des entstandenen Produktes eine Function der Menge des Fermentes, nicht aber der Menge der oxydirenden Substanz.

2. $\mathrm{Zu}$ den günstigsten Bedingungen der 0xydasewirkung gehört schwach alkalische Reaction, wie schon Bertrand und Bourquelot gefunden haben.

3. Die Laccase kann auf Grund ihres Stickstoff- und Schwefelgehaltes und ihrer Reactionen zu den Eiweisskörpern gerechnet werden. Ihr Aschegehalt kann sehr gering sein. ohne einen Einfluss auf die Oxydationswirkung zu haben.

4. Die Laccase wird weder durch schwache Säuren, noch durch peptische oder pancreatische Verdauung zerstört.

\section{Litteratur.}

1) Bertrand, C. R. de l'Ac. de Sc., 1894, S. 1215; ibidem 1895, S. 26 t und S. 1166; ibidem 1896, S. 1132.

2) Hikorokuro Yoshida, Journ. of the chem. Society, 1883.

3) Bertrand, C. R. de l' Ac. d. Sc., 1896, S. 1215, S. 463.

4) Bertrand und Bourquelot, G. R. d. l'Ac. d. Sc., 1895, S. 738.

5) Bourquelot, C. R. d. l'Ac. d. Sc., 1896, S. 260 u. 423.

6) Bouffard, C. R. d. l'Ac. d. Sc., 1897 S. 706 u. 1053.

7) Cazeneuve, C. R. d. l'Ac. d. Sc., 1897 S. 406; ibidem S. 781.

8) Laborde, C. R. d. l'Ac. d. Sc., 1897, S. 248-536; ibidem 1896 S. 1074.

9) Lagatu, C. R. d. l'Ac. d. Sc., 1897, S. 512.

10) Martinaud, C. R. d. l'Ac. d. Sc., 1895, S. 502.

11) Tolomei, Olease, Atti Accad. dei Lincei, 1896.

12) Boutroux, Le pain.

13) Duclaux, Traité de microbiologie, T. III, 1900.

14) Medwedew, Pflüger's Archiv, Bd. LIV.

15) Walther, Petersburg, Inaug.-Diss., 1898 (russ). 